\title{
A relação entre a dependência de cuidados, risco e úlcera por pressão*
}

Recebido em: 19/04/2012

Aceito em: 27/09/2012

\author{
Janete de Souza Urbanetto \\ Maria Angélica Casa Nova dos Santos ${ }^{2}$ \\ Angeline Francines Poltozi ${ }^{2}$ \\ Ana Lúcia Comparsi Pechansky² \\ Graziela $\mathrm{Hax}^{3}$ \\ Aline Custódio 4
}

Estudo com objetivo de analisar a associação entre os escores de cuidados do Sistema de Classificação de Pacientes (SCP) com as classificações de risco da Escala de Braden e a ocorrência de úlcera por pressão (UP). Delineamento transversal, com 2.562 pacientes adultos hospitalizados. Foram encontradas associações significativas dos cuidados intermediários e semi-intensivos do SCP e do risco elevado/moderado da Braden com a ocorrência de UP. Conclui-se que a utilização de instrumentos de avaliação dos pacientes são ferramentas valiosas para a prevenção de UP.

Descritores: Úlcera por Pressão, Classificação, Escalas, Segurança do Paciente.

\section{The relationship between care dependency and risk of pressure ulcers}

This study aims to analyze the association between scores for the care of Patient Classification System (PCS) with the risk ratings of the Braden Scale and the occurrence of pressure ulcers (PU). Cross-sectional design, with 2562 patients hospitalized adults. Significant associations were found for intermediate care and semi-intensive SCP and the high/moderate risk Braden with the occurrence of PU. It is concluded that the use of tools for evaluating patients are valuable tools for the prevention of pressure ulcers.

Descriptors: Pressure Ulcer, Classification, Scales, Patient Safety.

\section{La relación entre la dependencia de cuidados y el riesgo de úlceras por presión}

Se trata de un estudio cuyo objetivo es examinar la asociación entre las puntuaciones para el cuidado del Sistema de Clasificación de Pacientes (SCP) con las calificaciones de riesgo de la Escala de Braden y la aparición de úlceras por presión (UPP). Diseño transversal, con 2562 pacientes adultos hospitalizados. Se encontraron asociaciones significativas para los cuidados intermedios y semi-intensivo de SCP y el alto/moderado riesgo Braden, con la ocurrencia de UP. Se concluye que el uso de herramientas para la evaluación de los pacientes son herramientas valiosas para la prevención de úlceras por presión

Descriptores: Úlcera por Presión, Clasificación, Escalas, Seguridad del Paciente.

\section{INTRODUÇÃO}

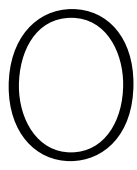
planejamento da assistência de enfermagem a pacientes internados tem sido cada vez mais orientado pela utilização de escalas ou diretrizes que identifiquem as condições dos pacientes, podendo contribuir para a qualidade e eficiência da assistência prestada. Um ponto importante para a obtenção de ótimos resultados é a identificação precoce de riscos à saúde dos pacientes.

O Programa de Segurança do Paciente da Organização Mundial da Saúde (OMS) define áreas prioritárias de atuação, com vistas à qualidade da assistência e segurança do paciente. Dentre elas está a prevenção de úlceras por pressão(1).

A UP é toda lesão na pele e/ou nos tecidos subjacentes, geralmente desenvolvida sobre uma proeminência óssea, como resultado da pressão isolada ou da pressão em combinação com a friç̧ão e/ou cisalhamento(2).

A Escala de Braden, criada em $1987^{(3)}$ e traduzida para o português em $1999^{(4)}$, tem sido considerada adequada na avaliação de risco para UP pela sua facilidade operacional na avaliação de seis aspectos: percepção sensorial, umidade, atividade, mobilidade, nutrição, fricção e cisalhamento. A soma da pontuação de cada aspecto remete à classificação de risco para UP, no qual a soma $\geq 16$ pontos caracteriza risco baixo, a soma de 11 a 16 indica risco moderado, e < 11 aponta alto risco(5).

O Sistema de Classificação de Pacientes $(\mathrm{SCP})^{(6)}$ é utilizado para avaliar a dependência dos pacientes internados para o cuidado de enfermagem por meio de 13 indicadores críticos: estado mental e nível de consciência, oxigenação, sinais vitais, nutrição e hidratação, motilidade, locomoção, cuidado corporal, eliminações, terapêutica, educação à saúde, comportamento, comunicação e integridade cutâneo-mucosa. A soma dos pontos para cada indicador remete a classe de cuidados ${ }^{(6)}$.

O objetivo deste estudo foi analisar a associação entre os escores de cuidados do SCP com as classificações de risco da Braden e a ocorrência de UP em pacientes adultos internados.

1 Enfermeira. Doutora em Ciências da Saúde pela PUCRS. Professora Adjunta do Curso de Enfermagem da PUCRS 2 Acadêmicas do Curso de Enfermagem da PUCRS.

3 Enfermeira. Mestre em Ciências da Saúde pela PUCRS. Integrante do Grupo de Cuidados da Instituição Hospitalar.

4 Enfermeira. Especialista em Enfermagem em Estomaterapia. Integrante do Grupo de Cuidados da Instituição Hospitalar.

*Trabalho de Conclusão de Curso de Graduação em Enfermagem da Faculdade de Enfermagem, Nutrição e Fisioterapia da PUCRS. 


\section{MÉTODO}

Estudo transversal realizado em um hospital universitário de Porto Alegre/RS. A população foi composta por adultos hospitalizados no ano de 2011, em unidades de internação, e a amostra totalizou 2.562 pacientes. Foram critérios de inclusão a idade $\geq 18$ anos, cada paciente possuir no mínimo duas avaliações no período de internação para a Braden e para o SCP. Quanto às avaliações, foram utilizadas a primeira (até $24 \mathrm{~h}$ de internação) e a última avaliação (antes da alta hospitalar ou óbito do paciente) relacionadas à Braden, ao SCP e à ocorrência de UP num total de 6.248 avaliações.

Os dados foram coletados por enfermeiros do referido hospital, capacitados para a avaliação de pacientes e a aplicação dos instrumentos. Buscou-se obter sociodemográficos (idade e sexo), de saúde (tempo de hospitalização, presença de UP e o desfecho da internação - alta ou óbito) e as pontuações de cada um dos instrumentos.

As pontuações da Braden foram categorizadas em Risco Moderado/Elevado ( $<16$ pontos) e Risco Baixo/Ausente $(\geq 16$ pontos) ${ }^{(5)}$. O SCP foi classificado em cuidados mínimos (13 a 26 pontos), cuidados intermediários (27 a 39 pontos), cuidados semi-intensivos (40 a 52 pontos) e cuidados intensivos (53 a 65 pontos) $)^{(5)}$.

A análise dos resultados ocorreu pela estatística descritiva e para a comparação de proporções entre as categorias de uma mesma variável foi utilizado o teste Qui-quadrado ou o teste Exato de Fisher, com simulação de Monte Carlo. Na busca por identificar os fatores relacionados à presença de UP, foi utilizado o modelo de regressão logística binária, com o objetivo de estimar diretamente as chances de ocorrência do agravo à saúde. Para a regressão logística ajustada, foram consideradas todas as variáveis com nível mínimo de significância inferior a 0,250 . Os dados receberam tratamento estatístico por meio do Software SPSS ${ }^{\circledR}$ versão 17.0 .

A pesquisa foi aprovada pelo Comitê de Ética em Pesquisa da PUCRS (OF.CEP-1.315/09 e 1.152/07) e foi assinado o termo de compromisso com o uso dos dados.

\section{RESULTADOS}

Dos 2.562 pacientes avaliados, houve uma concentração de pacientes do sexo feminino ( $n=1383 ; 54 \%)$ e da faixa etária de 60 anos ou mais $(n=1405 ; 44,8 \%)$. Quanto à hospitalização, o tempo de permanência prevalente foi entre 6 a 10 dias ( $n=829 ; 32,4 \%), 94$ pacientes $(3,7 \%)$ desenvolveram UP e a prevalência de óbitos foi de $4,2 \%$ ( $n=108)$.

As duas escalas estão retratadas quanto à sua pontuação e classificação, tanto na internação quanto na alta. O SCP teve pontuação média de 20,8 \pm 8,07 (mínima 13 e máxima 50) na internação, sendo que os pacientes foram classificados em $76,2 \%$ como de cuidados mínimos, 19,9\% cuidados intermediários e $3,9 \%$ cuidados semi-intensivos. Na alta, a pontuação média foi de $20,7 \pm 8,35$ (13 a 52), com 77,8\% de cuidados mínimos, $17,6 \%$ cuidados intermediários e 4,6\% cuidados semi-intensivos.

A pontuação média da Braden na internação foi 19,71 \pm 3,24 (7 a 23) e os pacientes foram classificados em $83,2 \%$ como de Risco Moderado/Elevado e 16,8\% como de Risco Baixo/Ausente. Já na alta, a pontuação média da Braden foi 19,84 \pm 3,46 (6 a 24) e os pacientes foram classificados em $84,5 \%$ como de Risco Moderado/ Elevado e 15,5\% como de Risco Baixo/Ausente.

A Tabela 1 demonstra que as pontuações da Braden e as do SCP estão associadas $(p \leq 0,01)$. Com relação à classificação das escalas, tanto na internação quanto na alta, foi constatada a prevalência de pacientes com cuidados mínimos (76,2\% e 77,8\%, respectivamente) no SCP e com Risco Baixo/Ausente para o desenvolvimento de úlceras por pressão (83,2\% e 84,5\%) pela Braden.

Ficou evidente que os pacientes que se situaram na classificação de Risco Moderado/Elevado para UP tinham maior prevalência na classificação de cuidados semi-intensivos. Sendo que esse padrão se manteve tanto na avaliação da internação quanto na da alta (Tabela 1).

A Tabela 2 apresenta a associação das variáveis de exposição do estudo com a ocorrência de UP. Quanto aos instrumentos avaliados, tanto as classificações do SCP quanto as da Braden estão associadas $(p \leq 0,01)$, sendo que a prevalência de pacientes com UP concentrou-se nas classificações de cuidados intermediários e semi-intensivos no que diz respeito ao SCP e na

Tabela 1 - Associação das classificações do SCP com as da Escala de Braden. $n=2.562$ pacientes.

\begin{tabular}{|c|c|c|c|c|c|}
\hline \multirow{2}{*}{\multicolumn{2}{|c|}{ Escalas }} & \multicolumn{2}{|c|}{ Braden na Internação } & \multicolumn{2}{|c|}{ Braden na Alta } \\
\hline & & $\begin{array}{l}\text { Risco Moderado/ } \\
\text { Elevado }\end{array}$ & Risco Baixo/Ausente & $\begin{array}{l}\text { Risco Moderado/ } \\
\text { Elevado }\end{array}$ & Risco Baixo/Ausente \\
\hline \multicolumn{2}{|l|}{$\mathbf{p}$} & \multicolumn{2}{|c|}{$\leq 0,01 €$} & \multicolumn{2}{|c|}{$\leq 0,01 €$} \\
\hline \multirow{3}{*}{$\begin{array}{l}\text { SCP na } \\
\text { Internação }\end{array}$} & Mínimos & $116(5,9)$ & $1836(94,1)$ & $125(6,4)$ & $1826(93,6)$ \\
\hline & Intermediários & $226(44,2)$ & $285(55,8)$ & $192(37,6)$ & $319(62,4)$ \\
\hline & Semi-Intensivos & $89(89,9)$ & $10(10,1)$ & $79(79,8)$ & $20(20,2)$ \\
\hline \multicolumn{2}{|l|}{$\mathbf{p}$} & \multicolumn{2}{|c|}{$\leq 0,01 €$} & \multicolumn{2}{|c|}{$\leq 0,01 €$} \\
\hline \multirow{3}{*}{ SCP na Alta } & Mínimos & $149(7,5)$ & $1843(92,5)$ & $90(4,5)$ & $1901(95,5)$ \\
\hline & Intermediários & $192(42,6)$ & $259(57,4)$ & $213(47,2)$ & $238(52,8)$ \\
\hline & Semi-Intensivos & $90(75,6)$ & $29(24,4)$ & $93(78,2)$ & $26(21,8)$ \\
\hline
\end{tabular}

n(\%); €Pearson Qui-Quadrado. 
Tabela 2 - Associação das variáveis sociodemográficas, de saúde, do SCP e da Braden com a ocorrência de UP durante a hospitalização. $\mathrm{n}=2.562$ pacientes.

\begin{tabular}{|c|c|c|}
\hline \multicolumn{3}{|c|}{ Presença de UP } \\
\hline & Não $(n=2.468) \mid$ & $\operatorname{sim}(n=94)$ \\
\hline SCP (cuidados)* & \multicolumn{2}{|c|}{$\leq 0,001 €$} \\
\hline Mínimos & $1981(80,3)$ & $11(11,7)$ \\
\hline Intermediários & $409(16,6)$ & $42(44,7)$ \\
\hline Semi-Intensivos & $78(3,2)$ & $41(43,6)$ \\
\hline Escala de Braden (r & \multicolumn{2}{|c|}{$\leq 0,001 \epsilon$} \\
\hline Baixo/Ausente & $2151(87,2)$ & $14(14,9)$ \\
\hline Moderado/Elevado & $316(12,8)$ & $80(85,1)$ \\
\hline Sexo & \multicolumn{2}{|c|}{$0,187 €$} \\
\hline Masculino & $1142(46,3)$ & $37(39,4)$ \\
\hline Feminino & $1326(53,7)$ & $57(60,6)$ \\
\hline Idade (anos) & \multicolumn{2}{|c|}{$\leq 0,001 £$} \\
\hline Até 19 & $12(0,5)$ & $0(0,0)$ \\
\hline $20-40$ & $480(19,4)$ & $5(5,3)$ \\
\hline $41-59$ & $651(26,4)$ & $9(9,6)$ \\
\hline$\geq 60$ & $1325(53,7)$ & $80(85,1)$ \\
\hline \multicolumn{3}{|c|}{ Tempo de Hospitalização (dias) $\quad \leq 0,001 €$} \\
\hline Até 5 & $577(23,4)$ & $7(7,4)$ \\
\hline 6 a 10 & $805(32,6)$ & $24(25,5)$ \\
\hline 11 a 15 & $416(16,9)$ & $12(12,8)$ \\
\hline$\geq 16$ & $670(27,1)$ & $51(54,3)$ \\
\hline Desfecho & \multicolumn{2}{|c|}{$\leq 0,001 €$} \\
\hline Alta & $2382(96,5)$ & $72(76,6)$ \\
\hline Óbito & $86(3,5)$ & $22(23,4)$ \\
\hline
\end{tabular}

$£$ Exato de Fisher; $€$ Qui-Quadrado.

* Utilizada a média resultante das pontuações da internação e da alta.

classificação de Risco Moderado/Elevado da Braden.

Também houve associação estatística $(p \leq 0,01)$ com a idade de $\geq 60$ anos e com as variáveis tempo de hospitalização e desfecho.

No intuito de identificar a existência de fatores confundidores na associação entre as variáveis estudadas e a ocorrência de UP, foi aplicada a regressão logística binária. Nessa análise permaneceram associadas apenas as classificações do SCP para cuidados intermediários (10 vezes mais chances de desenvolverem UP) e semi-intensivos (39 vezes mais chances de desenvolverem UP) quando comparados à classificação de cuidados mínimos; e para a classificação de Risco Moderado/Elevado para UP da Braden, com 5,8 vezes mais chances de desenvolvimento de UP se comparado aos pacientes na classificação Risco Baixo/Ausente (Tabela 3).

Apesar de a idade ter se associado à ocorrência de UP $(p \leq 0,001)$, a mesma não foi analisada na repressão logística em função de apresentar caselas com número de pacientes abaixo de cinco.

\section{DISCUSSÃO}

Este estudo encontrou associação do SCP (cuidados intermediários e semi-intensivos) e da Braden (Risco Moderado/Elevado) com a ocorrência de UP. Ainda constatou que as duas escalas estão associadas entre si, confirmando que os pacientes com classificação de cuidados semi-intensivos no SCP concentram-se na classificação de Risco Moderado/Elevado para o desenvolvimento de UP. Outro aspecto importante é que os pacientes em cuidados intermediários distribuem-se de forma semelhante com prevalência maior na classificação de Risco Baixo/Ausente para o desenvolvimento de UP, tanto na internação quanto na alta.

Não foram encontrados na literatura artigos que associem essas duas escalas de avaliação, dessa forma elas serão discutidas separadamente.

O escore médio da pontuação para a Braden, neste estudo, foi de 19,9 $\pm 3,0$. Estudo realizado em unidades de internação e de intensivismo encontrou escore médio inferior (13,8 $\pm 4,7$ pontos) (7), provavelmente por se tratarem de pacientes com agravos maiores à sua saúde.

O SCP teve um escore médio da pontuação de $21,0 \pm 7,4$, o que classifica uma prevalência dos pacientes em cuidados mínimos. Um estudo realizado em unidades de internação cirúrgica também encontrou prevalência dos pacientes em cuidados mínimos $(41,7 \%)^{(8)}$. Outro estudo constatou em escore médio mais elevado $(31,9 \pm 4,8$ pontos) quanto ao SCP em pacientes atendidos em uma unidade de hemodinâmica, com prevalência dos pacientes em cuidados intermediários ${ }^{(9)}$.

Outro estudo que avaliou o SCP internados em pronto-socorro mostrou que $44,4 \%$ dos pacientes foram classificados como de cuidados intermediários e $38,2 \%$ em cuidados mínimos ${ }^{(10)}$, o que diferencia dos achados deste estudo.

Com relação ao tempo de hospitalização e a ocorrência de UP, a permanência hospitalar superior a 15 dias concentrou $54,4 \%$ dos

Tabela 3 - Chances para o desenvolvimento de UP durante a hospitalização, conforme regressão Logística Ajustada.

\begin{tabular}{|c|c|c|c|}
\hline \multicolumn{4}{|c|}{ Presença de UP } \\
\hline & $\mathbf{p}$ & OR & IC $-95 \%$ \\
\hline \multicolumn{4}{|l|}{ SCP (cuidados)* } \\
\hline Mínimos & & $\ldots . .$. & $\ldots . .$. \\
\hline Intermediários & $<0,001$ & 10,509 & $4,337-25,463$ \\
\hline Semi-Intensivos & $<0,001$ & 39,716 & $14,212-110,926$ \\
\hline \multicolumn{4}{|c|}{ Escala de Braden (risco) } \\
\hline Baixo/Ausente & & $\ldots \ldots$. & $\ldots . .$. \\
\hline Moderado/Elevado & $<0,001$ & 5,826 & $2,923-11,6098$ \\
\hline \multicolumn{4}{|l|}{ Sexo } \\
\hline Masculino & & ....... & ....... \\
\hline Feminino & 0,163 & 1,424 & $0,866-2,340$ \\
\hline \multicolumn{4}{|c|}{ Tempo de Hospitalização (dias) } \\
\hline Até 5 & & ....... & ....... \\
\hline 6 a 10 & 0,838 & 1,102 & $0,434-2,800$ \\
\hline 11 a 15 & 0,551 & 0,730 & $0,259-2,066$ \\
\hline$\geq 16$ dias & 0,071 & 2,246 & $0,934-5,405$ \\
\hline \multicolumn{4}{|l|}{ Desfecho } \\
\hline Alta & & ....... & .......... \\
\hline Óbito & 0,699 & 1,132 & $0,603-2,124$ \\
\hline
\end{tabular}

Regressão Logística ajustada para SCP, Braden, Sexo e Tempo de Hospitalização e desfecho. 
pacientes que desenvolveram UP. Outro estudo também encontrou associação entre o tempo de internação e o risco elevado para o desenvolvimento de UP em $95 \%$ dos pacientes com tempo superior a 15 dias $^{(7)}$. Esses dados são reforçados por outra pesquisa que encontrou associação $(p=0,015)$ entre o tempo de permanência em torno de 18,4 dias e a ocorrência de UP ${ }^{(11)}$.

Foi encontrada associação da ocorrência de UP, na avaliação inicial, com o tempo de hospitalização, as classificações do SCP (cuidados semi-intensivos e intermediários) e da Braden. No entanto, quando utilizou-se regressão logística, apenas as classificações do SCP para cuidados semi-intensivos e intermediários e a classificação de Risco Moderado/Elevado da Braden estiveram associadas à ocorrência de UP, com chances maiores se comparadas aos pacientes nas categorias de referência dos dois instrumentos. Outro estudo também encontrou chances para o desenvolvimento de UP 3,8 vezes maior em pacientes com risco moderado e 7,3 vezes maior em pacientes com risco elevado na classificação da Braden se comparado aos pacientes classificados como sem risco para UP(7).

Outro aspecto relevante na avaliação com foco na prevenção é a continuidade da avaliação. É necessário que a avaliação seja sistematizada e periódica mesmo após o aparecimento de UP. Este estudo encontrou uma prevalência baixa de UP (3,7\%), mas demonstrou que a prevalência de pacientes que desenvolveram mais de uma UP durante a internação foi de $39,4 \%$. Esse aspecto é reforçado em outro estudo que relata que a continuidade das avaliações para identificação do risco de UP pode prevenir a ocorrência de úlceras em outros locais ${ }^{(7)}$. Outros estudos realizados encontraram incidência muito superior, com taxas de 26,8\%, 32,1\% e $39,8 \%$, respectivamente ${ }^{(12-14)}$. Ainda, outro estudo que avaliou 78 pacientes com UP, identificou que $68 \%$ destas ocorreram durante a internação( ${ }^{(15)}$.
Os resultados comprovam que existe uma relação entre dependência e/ou risco para UP e a ocorrência de UP e que a utilização de instrumentos de avaliação podem facilitar a identificação do risco e o estabelecimento precoce de medidas de prevenção de UP. No entanto, embora exista a necessidade de implantação de uma avaliação sistematizada dos pacientes, na prática cotidiana dos profissionais essa realidade ainda é pouco encontrada. Estudo realizado para avaliar o conhecimento dos profissionais de enfermagem sobre a prevenção de UP mostrou que $20,6 \%$ dos enfermeiros e $26,4 \%$ dos auxiliares/técnicos de enfermagem apresentavam déficits de conhecimento em algumas áreas referentes ao tema ${ }^{(16)}$.

\section{CONSIDERAÇÕES FINAIS}

Os dados do estudo permitiram responder ao objetivo deste estudo, no qual foi encontrada relação estatística significativa $(p<0,001)$ entre as classificações do SCP da Braden. Também permitem afirmar que os pacientes classificados como intermediários e semi-intensivos pelo SCP e com Risco Moderado/Elevado pela Braden têm chances maiores de desenvolver UP, se comparados às outras classificações dessas escalas. Ocorreu aumento na prevalência de pacientes em escores de dependência maior e Risco Moderado/Elevado para UP por ocasião da alta.

Os dados encontrados neste estudo reforçam que a utilização de instrumentos de avaliação dos pacientes são ferramentas importantes para o planejamento em saúde.

A limitação deste estudo foi o número reduzido de pacientes com UP se comparados com a amostra, o que traz limitações para a análise dos dados e pode, na regressão logística, estar superestimando as chances de ocorrência de UP. No entanto, os dados permitem afirmar que é possível e indicada a utilização desses instrumentos para a avaliação de riscos para o desenvolvimento de UP em pacientes internados.

\section{Referências}

1. Organização Pan Americana da Saúde. Brasil Boletins Informativos da OMS para

Segurança do Paciente [Internet]. Disponível em: http://new.paho.org/bra

2. European Pressure Ulcer Advisory Panel and National Pressure Ulcer Advisory

Panel. Prevention and treatment of pressure ulcers: quick reference guide [Internet].

Disponível em: http://www.epuap.org/guidelines.html.

3. Bergstrom N, Braden BJ, Laguzza A, Holman V. The Braden Scale for predicting

pressure sore risk. Nurs Res. 1987;36(4):205-10.

4. Paranhos WY, Santos VLCG. Avaliação de risco para úlceras de pressão por meio da escala de Braden, na língua portuguesa. Rev Esc Enferm USP. 1999;3(Esp):191-206.

5. Maklebust J, Sieggreen M. Pressure ulcer: guidelines for prevention and nursing

management. Pennsylvania: Springhouse Corporation; 2000.

6. Perroca MG, Gaidzinski RR. Sistema de classificação de pacientes: construção e

validação de um instrumento. Rev Esc Enferm USP. 1998;32(2):153-68.

7. Gomes FSL, Bastos MAR, Matozinhos FP, Temponi HR, Meléndez GV. Avaliação de risco

para úlcera por pressão em pacientes críticos. Rev Esc Enferm USP. 2011;45(2):313-8.

8. Fugulin FMT, Gaidzinski RR, Kurcgant P. Sistema de classificação de pacientes:

identificação do perfil assistencial dos pacientes das unidades de internação do HU-

USP. Rev Latinoam Enferm. 2005;13(1):72-8.

9. Hammermüller A, Rabelo ER, Goldmeier S, Azzolin KO. Classificação de pacientes

atendidos em unidade hemodinâmica segundo o grau de dependência dos cuidados de enfermagem. Acta Paul Enferm. 2008;21(1):72-6.

10. Zimmermann LP, Magnago TSBS, Urbanetto JS, Greco PBT, Viero NC, Vieira TG,

Ceron MDS. Avaliação do grau de dependência de cuidados de enfermagem dos

pacientes internados em pronto-socorro. Rev Enferm UFSM. 2011;1(2): 153-63.

11. Fernandes LM, Caliri MHL. Uso da Escala de Braden e de Glasgow para

identificação do risco para úlceras de pressão em pacientes internados em centro de

terapia intensiva. Rev Latinoam Enferm. 2008;16(6):973-8.

12. Sousa CA, Santos I, Silva LD. Aplicando recomendações da Escala de Braden e prevenindo úlceras por pressão - evidências do cuidar em enfermagem. Rev Bras Enferm. 2006;59(3):279-84.

13. Hans M, Bitencourt JVOV, Pinheiro F. Fatores de risco adicionais à Escala de Braden: um risco para úlceras de pressão. Enferm Foco. 2011;2(4):222-5.

14. Rogenski NMB, Santos VLCG. Incidence of pressure ulcers at a university hospital. Rev Latinoam Enferm. 2005;13(4):474-80.

15. Blanes I, Duarte IS, Calil JA, Ferreira IM. Avaliação clínica e epidemiológica das úlceras por pressão em pacientes internados no Hospital Ssão Paulo. Rev Assoc Med Bras. 2004;50(2):182-7.

16. Miyazaki MY, Caliri MHL, Santos CB. Conhecimento dos profissionais de enfermagem sobre prevenção da úlcera por pressão. Rev Latinoam Enferm. 2010;18(6):1203-11. 